\title{
A CASE OF CARCINOMA OF THE MIDDLE EAR, PROBABLY ENDOTHELIOMA.
}

By W. Sohier Bryant, A. M., M. D.

NEW YORK.

The patient was a man 41 years old; very fat, extremely nervous and painfully apprehensive. The previous history was acquired for the most part at intervals throughout the course of the disease. At the first visit the evidence pointing to malignant growth was nil, save for the unusual appearance of a polyp in the external canal. The weight of evidence afforded by a study of the symptoms favored a diagnosis of ordinary chronic suppuration of the middle ear with acute involvement of the mastoid cells. The patient said that he had a discharging ear in childhood following measles, but could give no definite account of subsequent aural difficulty until the onset of the present trouble. He had eaten and drunk excessively for many years, but had kept himself in very good physical health by constant out-of-door exercise. For the past five years he had noticed that the right eyebrow was somewhat lower than the left. $\mathrm{He}$ admitted the existence of a primary luetic lesion some years previous to the time when he consulted me. His brother thought that the patient had been peculiar mentally for two years or more. About a year ago the patient found that he did not hear well, and that there was a pulsation in the left ear. He had a cold in the head at that time. His brother noted that the patient had spilled a great deal of food over his clothing for some time past. The patient ten months ago first noticed pain at the top of the head. Seven months ago he noticed that the left ear was covered with "pimples" (probably herpes), which persisted for ten weeks. He remembered that he had shooting pains in and around the left ear and mastoid process, together with ringing noises six and one-half months ago. Six months ago, headaches and twitching of the face began. Three months ago the patient had severe pains in left lower bicuspids. These were pulled out and found to be perfectly normal. Very little pain was 
caused by the tooth extraction, but the lower jaw felt numb. The pain in the jaw persisted. About that time he had an attack of bronchitis, accompanied by the pouring out. of an abnormal amount of thick mucus from the left side of the nose, and pain and discharge in the left ear. Two months ago he first noticed that he had double vision, a condition confirmed by Dr. Arnold Knapp at about this time. Six weeks ago the patient noted that he could not hear in the left earr. A month ago facial paralysis was noticeable. Two weeks ago the patient noted that left side of forehead did nut wrinkle.

Family History.-The patient's mother and two maternal aunts, died of cancer of the breast. One of his sister's sons, a young man, died of malignant disease of the intestines.

Examination.-A hard, flat polypoid granulation filled the fundus of the meatus. It had an unnatural look, and was smoother, harder, flatter and paler than a true granulation, and apparently was attached to the promontory. It was not sensitive. There was a slight tenderness of the mastoid process. A fork having 512 single vibrations, when placed on the glabella, was referred to the left ear. Patient was very deaf in the left ear; he could hear no fork by air conduction below 51 ? single vibrations. Bone conduction was fair. The nose was not much obstructed save by slight hypertrophy and thickening of the septum on the left. $\mathrm{He}$ thought his sense of smell on the left was not as good as it used to be, and that the saliva did not flow as well on that side as formerly. His roice was a little thick. Tongue deviated to left. No dizziness. Pain in top of head and cheek.

Dr. 'Ward A. Holden examined the eyes. He found the left pupil sluggish and slightly irregular. The fundi ociuli were normal. $V$ ision 20/15ths each. Double vision, images horizontal; diminished sensation of face; paralysis of left external rectus; knee jerk exaggerated.

Dr. George S. Dixon made a blood examination and found: Red.cells, 5,540,000; white cells, 8,200; hemoglobin, 90 per cent.

The differential count was as follows:

Small mononuclear.......36.6 per cent.

Large mononuclear....... 7.8 per cent.

Transitional . . ......... 3.4 per cent.

Polynuclear . . . . . . . . . 51.2 per cent.

Eosinophile .......... .4 per cent. 


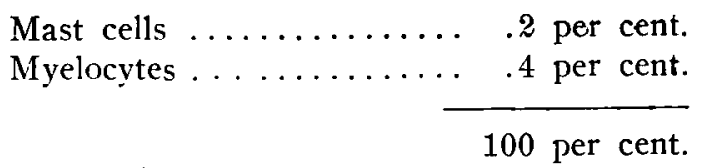

At this time the pulse was 100 and the temperature $100.5^{\circ}$ F. by mouth.

When first seen on August 2, 1906, the writer made a diagnosis of otitis media purulent chronica of the left ear with slight mastoiditis and nerve complications, but on the following day he changed it to syphilis of the ear because of the atypical polyp. The patient received the usual treatment for chronic suppuration of middle ear. Later on iodid of potassium was given.

During the second week of observation, the patient would not be controlled in any way. He could not sleep without twenty grains of veronal and one grain of codein at night. The left ear showed only a little serous discharge in the meatus. The polypus appeared to fill the tympanus and was most in evidence above and in front. Pulse, 80 ; temperature, $98.6^{\circ}$. There was dull pain in the temporal region, behind and in front of the ear and also in the occiput. After removal of some fragments of the growth with forceps, the stump came down and replaced the portion which had been removed. The diagnosis of malignant growth was now made, most probably sarcoma. because of the hardness.

Dr. George S. Dixon reported that a stained section from the specimen removed was negative. He said that the curettings submitted showed a mass of crystalline material-inclefined in form, and hence unrecognizable - possibly cholesterin, together with general detritus such as one would expect to find in a chronic suppurating middle ear. In addition some granulation and new-formed connective tissue were present. He found nothing to indicate malignant disease, or lues. He was of the opinion that (if cholesteatoma could be ruled out) the patient had an ordinary chronic suppurative otitis with the formation of granulation tissue, some of which under favorable'conditions may be converted into connective tissue.

Third Week of Obserzation.-Patient had pain above the left ear in temporal region. Voice thick. Inner end of canal seems to be contracting. Facial paralysis quite noticeable. Dr. Edwin G. Zabriskie noted the left pupil did not react as quickly to light as did the right, and that the patient could 
not open left side of mouth as wide as right. The patient heard the tuning fork (5\%2 S. V.) on the right mastoid process and referred it to the left ear. Paresis of facial movements least in frontalis muscle. Pulsating tinnitus. Watch not heard in left ear by air or bone. Probing growth causes pain in gums on left. Some occiptal pain. Growth has entirely filled fundus of meatus since the curettement and has increased chiefly anteriorly.

Dr. E. G. Zabriskie's E.ramination.-Complete loss of sense of smell. Sensory innervation of face all right except left lower angle of mouth, which reacts to a prick only.

Fourth Week of Observation.-Paralytic symptoms much increased. External rectus absolutely paralyzed. Tongue is markedly deviated to left. 'The left eye closes voluntarily, but with difficulty. It does not wink. He can not whistle. Pains largely. occipital and parietal as before. There is no feeling in temporal region back of eye. Paralysis of sensation most marked under eye. Hemianesthesia of head and considerable redness. Fork 512 single vibrations heard slightly by air conduction in left ear. Bone conduction distinctly better in left ear than in r:ght. Touches tip of nose with tip of torefingers readily. with shut eyes. Cerebration a little slow.. The growth was wiped with 1-500 bichlorid of mercury, which has a retarding effect on it. It is lobulated and slightly larger than before. Muscles of mastication do not contract on left side. At this time the growth was snared and curetted and all diseased tissue that could be seen was removed. It was sessile and hard, especially in its anterior portion. The membrana tympani and malleus when uncovered appeared intact except where the membrane was torn in front by the manipulations. The growth apparently sprang from the anterior portion of the annulus tympanicus.

The bone of the inner end of the meatus and tympanic was scraped. It appeared sound. After the curettage the meatal walls were hard and intact. The walls of the inner, anterior and upper tympanum could not be examined on account of the ossicles and membrane. A few hours later the growth had been pushed out, obliterating part of inner end of canal. The end of the stump was ragged, showing that the growth had been extruded and not propagated at its periphery.

Dr. Edward G. Zabriskie's report of the histologic examination of the second specimen removed said that the 
granulations showed a faintly strained stroma of compact connective tissue fibres and irregularly scattered throughout this, more particularly in the lymph spaces, were groups of round cells closely packed together, which in certain places appeared to be springing from the endothelial lining of these spaces. These cells, the nature of which it was rather difficult to determine, because of their poor nutrition and consequently poor straining capacity, appeared to be crowding along the lymph channels. In some instances they were carried along by the stream or pushed from behind and in others sprang directly from the walls of the channels. They were most probably invading masses from the central growth which was as yet quite inacessible. There were no direct evidences of a specific granuloma in any of the sections that he examined, nor were there any of the definite vascular changes that one would have expected to find in this condition. $\mathrm{He}$ considered these sections as indicative of a malignant growth, and was rather inclined to consider it an endothelioma. Of course, it was quite impossible to state the origin of the growth from the evidence at hand. Dr. Zabriskie found some cartilage in the growth, which was probably a portion of the orbicular cartilage.

Dr. George S. Dixon reported that the last tissue submitted from the canal showed that the operation had been more successful in obtaining a portion of the tumor. The fragments indicated the presence of carcinoma.

The writer had the privilege of examining the sections made by both Dr. Dixon and Dr. Zabriskie, and was convinced of the correctness of their observations.

Following the removal, the patient thought his ear better, but other head symptoms worse. Valsalva's inflation negative. Walks a little unsteadily in the dark. Speech very thick. Can't drink without spilling at corner of mouth. Eye does not quite close. Left middle turbinate close to septum, but shrinks with adrenalin.

Fifth Week of Observation.-Left ear hears the low forks faintly by air. Upper forks heard chiefly by right ear.

Dr. Ward A. Holden reported pupils normal, but found a neuroparalytic keratitis.

Dr. J. Ramsey Hunt saw the case again and reported the cranial nerve symptoms unchanged. He referred disturbance of taste to the trigeminus rather than to the chorda tympani, because patient could not detect salt, sweet and sour 
on the left. The palate was distinctly paretic on the left. The iodid seemed not to have any effect. He advised mercury by inunction or hypodermatically.

The patient is unable now either to s'eep or to eat anything solid because of the pain. No pain in ear. There is great mental depression. Fork heard in left ear through bone from all parts of head. When the right meatus is closed with the finger he hears the fork by bone from all parts of the head only in the right ear. Pulsating tinnitus on the left. Annoying pain in the left eye, forehead, vertex, occiput and back of jaw, etc. A reflex light on growth shows a slight pulsation. Meatus fairly wide open, showing what appeared to be membrana tympani posteriorly and superiorly. Can't stand on one foot with eyes shut, but does so easi.y with eyes open.

Sixth Weck.-Codein 1-1/2 grains and 15 grains sulphonal at night. Pain less. Meatus now completely filled at inner end. Some necrosis caused by the local application of bichlorid of mercury. The growth was coming outward alone the anterior wall of the meatus. Crystal bichlorid of mercury applied to growth. More difficulty in talking and eating. Most of the pain was in lower part of forehead and about eye. Is still able to feel hard pressure on face. Paralysis of seventh nerve not quite so marked about eye. Left nares is becoming stopped with hard material. Patient said dizziness was increasing. Paralysis more marked.

Seventh Week.-Postrhinoscopic examination prevented by retraction of palate and exaggerated pharyngea reflex, garging and vomiting. Left nares stopped up posteriorly. Auditory meatus slowly filling from the fundus with the hard growth. Complained of more dizziness and double vision. The dizziness is probably from the double vision in eye. Convergent strabismus of left eye noticeable. Postnasal digital examination found the end of left inferior turbinate. A soft uneven surface lay above it, but was out of definite reach. Tears flow on left cheek. Metallic taste in mouth has been marked. Left pupil a little larger than right, it reacts to light and accommodation. Patient extremely fearful of death or operation. Dr. Holden could note no change in the eye.

Eighth Week.-Operation under ether. Made the usuai postaural mastoid incision. Removed the posterior, upper and anterior wall of the osseous meatus. Both bone and soft 
tissue with the malignant growth were stripped from the walls and the tympantum and Eustachian tube were cleaned. The dura mater of the middle and posterior fossae was fully exposed. It appeared perfectly normal. Washed out with cold saline; packed the meatus lightly with iodoform gauze. One deep suture and metal clamp external suture. The growth was not attached anywhere except in the fundus of the meatus anteriorly, and in the Eustachian tube. It did not appear to involve the bone. No lead could be found in any direction. The operation did not continue to the apex or the pyramid because the patient had absolutely refused to allow any intracranial exploration.

Ninth Week.-First day of convalescence. Changed outer dressings. Much depressed at times. Consultation with Dr. Charles $H$. Knight about swollen tongue from injury during anesthesia. Second day: Paralysis of facial and chorda tympani seemed better. Less metallic taste in the mouth. Less facial paralysis. Orbicularis of left eye acts strongly. Dressings only slight'y stained. Changed packing in the meatus. Removel the metal skin clamps. Wound healing by first in. tention. Third day: Wound firm and solid. Changed gauze in the meatus. Fourth day: No sign of suppuration in ear. Sixth day: Facial paralysis marked. Eye does not close. Pain has been bad again. The fundus of the canal nearly healed. It has collapsed *somewhat at bottom. Slight serous discharge. When the fundus of the ear was wiped, deep pain was felt in jaw at the place of the tooth extraction.

Tenth Week.-Seventh day of convalescence. Some pain in brachial plexus, relieved by chloroform liniment. A growth is visibie in the meatus on the inner ante. ior wall in about the former position. Removed the excrescences with forceps. Eighth clay: Slept well. Feels pretty well. In consultation Dr. C. L. Dana suggested a basal cranial radical operation. Growth in meatus spreading and meatus narrowing. Ninth day: The lumen of the meatus filling up with growth. The growth on floor of meatus moves with movement of jaw. No dizziness can be detected. Dizziness increases on moving about. Has a subjective feeling of pressure at the vertex. A cotton plug saturated in 1-1000 corrosive subimate worn in the meatus. A brawny swelling on junction of left hard and soft palates. Left nose still more occluded. Tenth day: Complains of feeling of weight on head. Consultation with Dr. Emil Gruening. He thinks growth located at apex of 
pyramid and that it could be removed. Twenty-five mgr. radium bromid of $1,000,000$ radio-activity exposed in meatus for thirty minues. Eleventh day: Radium forty-five minutes. Growth has considerably increased in meatus. Thirteenth day: Radium thirty minutes. Slept well. Ear looks a little smoother and cleaner. One two-hundredths bichlorid wiping. Iodid of potassium disturbed stomach and was discontinued.

Eleventh Wcck-Fourteenth Day.-Dr. E. D. Janeway consulted. He said that there was paralysis of the ninth nerve. Patient hears all tuning forks from all parts of the head in left ear. Fifteenth: Radium twenty-five minutes. Meatus filling in slowly with smooth hard masses. Transillumination shows large clear frontal sinus and antrum. The left antrum seems to glow more than right. Sixteenth day: General conclition better. Nasal breathing worse. Pain in gums of lower jaw and left side of nose. Seventeenth day: Face seems to show more motion. Occipito-frontalis moves a little. Adrenalin opens right nose completely; left nose shrinks anteriorly but not posteriorly. Pain chiefly in lower jaw, where tooth was pulled, a little also in nose and cheek. Radium fifty minutes. Ear seems to have closed little since last observation. Eighteenth day: Consulted Dr. Joseph Collins. $\mathrm{He}$ advised immediate radical operation. Ea: looks a little bet. ter. Double vision, sees an object to one side and above the other object. Ataxia slight. Nineteenth day. Radium seventy minutes. Morphin. Pupils large. Fundus of ear now open and pale. Twentieth day: Consultation with Dr. J. R. Shannon. He said that there was slight commencing optic neuritis. Corneal irritation was due to facial and trifacial paralysis, and to external applications for eye pain. Diminished visual field. External rectus the only eye muscle affected. Slight convergent strabismus. Subcutaneous use of bichlorid of mercury discontinued. Pain in face very bad last night. Pulse, 100; temperature, $99^{\circ}$.

Treelfth Weck.-Vomiting. Feels worse every day. Faul continues to increase in whole side of head, face and neck, temples and vertex. Eye and gum pain the most intense. Feeling of weight over left vertex. Pain not controlled by morphin. Almost sleepless. Voice quite husky. Left nasal fossa closing fast. Probe will not pass. Left pupil smaller than right, sluggish and reacts little. 
Thirteenth Weck.-Consultation with Drs. Harvey Cushing, Joseph Collins and J. B. Walker. Dr. Cushing said that he had operated on several similar cases. He thinks the ear growth had nothing to do with the paralysis; that the parày sis of fifth and sixth cranial nerves and the pain were due to pressure on the Gasserian ganglion; that the growth closing left nares has penetrated skull and lifted Gasserian ganglion and at same time pressed on seventh nerve. Thinks removal of Gasserian ganglion would relieve pain. Said the ophthalmic and pupillary disturbances are due to contiguity of the nerves, and that the lingual and facial paralysis are only apparent and are due to loss of muscular sense. Said optic disc showed changes. Patient complains of most pain in vertex and left side of head. Slept little and ate less. Occasionally. says the wrong word for things. Much vomiting.

Fourteenth Week.-Fundus of meatus filling slowly or collapsing. Pain in occiput. Worst pain in lower jaw and eye. constant. Eye red; exophthalmos, trophic conjunctivitis. Excoriation of left brow, nose, lip, chin and gums from picking with finger-nails. Losing weight fast. Nose more stopped, full of bloody mucus. Swallows into trachea. Ear unchanged. Consultation with Dr. F. H. Bosworth, who said there was paralysis of left recurrent laryngeal nerve and left vocal chord; that is, paralysis of left pneumogastric and glossopharyngeal nerves. Said there was paralysis of the left palati. Also an enlargement on the posterior end of lower turbinal.

Fifteenth Weck.-Failure of physical and mental strength is marked. Left nares constantly full of thick mucus, which is very annoying. as he cannot dislodge it. Since operation has had no pain in or about the ear; pain chiefly in eye, gum of lower jaw, in nose and side of nose. More pain now in vertex and occiput. Fundus of auditory meatus filling up slowly, probably collapsing. Soft palate as seen by mouth has an antero-posterior furrow with bulging on each side, most marked to left, hard on left side, soft on right. Patient often chokes from inhalation of saliva and food. Fear of choking makes him sleep in the erect position. Had a hard lump in left anterior supraclavicular region.

Sixteenth Week.-Ear remains dry. Palatal induration extending to right side. Pulse weaker, rate as usual. Ear filled up about as much as before operation. Physically, a little weaker, mind a little better ;ear, eye, nose and throat dry. 
Some enlarged glands under angle of left jaw. Thickening in palati region is increasing and extending. Pain in eye, gum, cheek, left parotid and lumbar region. Feels feverish.

Serenteenth Week-Nose still further occluded. Palate a little fuller. Has had "sticking" pains in vertex of left nose and a little in left ear. Gums of upper left molars ulcerating on inner side. No bone conduction in left ear. Tuning fork not heard on left mastoid, but is heard on right. Complains of dry throat. Left nares contains bloody, thick mucus. Left pupil smaller than right. Right pupil dilated. Says morphin does not stop pain unless it stupefies him, but it lessens it.

Nineteenth Weck.- Has had a large lump in lower carotid triangle for a week. Much severe pain, fifteen grains and more of morphin daily. Regurgitation through nose. Losing strength rapidly, weight not much changed.

Tuenty-second Weck.-Looks as if he had lost more" weight. Is very despondent. Twenty-five grains of morphin daily. Facial and other paralysis more marked. More exophthalmos. Hard lump over masseter muscle, left angle of jaw, below jaw and at level of cricoid cartilage. Palate growth larger, more prominent and red. Anteriorly, it is pale and shows dark brown ecchymosis. Speech more difficult and exhausting.

Trenty-fourth Week.-Had violent attack of dyspnea at stool. More than fifty grains of morphin daily. Pleuritic effusion on left.

The courtesy of Dr. C. R. Putnam, who had charge of the case toward the end, provides us with the following notes: The patient took large quantities of food. At first he swallowed into the trachea, because the throat was anesthetic, but he finally learned to swallow without difficulty. He had a metastatic growth in the mediastinum, which pressed the trachea to the right, causing orthopnea for several weeks before death. He had severe thoracic pains, took fifty grains of morphin daily. Atropin, strychnin and nitroglycerin were used when indication presented themselves.

Twenty-sixth Week of Observation.-January 21st: Died after three days of dyspnea. Did not get out of chair or go to bed for fear of choking. Used chloroform to ease severe dyspnea. Loss of weight had been slight of late. Weight about 200 , formerly about 230 . Death occurred from thoracic rather than from intraeanial causes. The ear had remained cicatrized to the last. Submaxillary lymph glands hard and as 
large as pigeon's egg. Swelling on ramus of left lower jaw. Largest single dose of morphin was twenty-six grains.

SUMMARY.

CHRONOLOGICAL SEQUENCE OF SYMPTOMS.

Running ear. Right eyebrow drooped. Mental change. Decrease of hearing in left ear and tinnitus. Pain in vertex. Herpes of the ear. Pain in and about the ear, headache, twitching of face. Severe pain in teeth and numbness of iaw, first noted trouble in the nose. Double vision. Total ieafness left ear. Marked facial paralysis.

ANALYSIS OF SYMP'TOMS.

The ear had been affected many years when mental changes were noted. Functional disturbances of the ear. Painful svmptoms in the vertex. Herpes of the ear indicating nerve lesions near the geniculate ganglion (Hunt). General headache and pain in and about the ear, functional disturbance of facial nerve. Functional disturbance of fifth nerve and disturbed nasal function. Disturbance of the external rectus.

ANATOMIC EFFHCTS.

We have followed the history of a cranial lesion first definitely affecting the function of the ear probably by encroachment on the tympanum near the orifice of the Eustachian tube: next it is located in the neighborhood of the geniculate ganglion: there is definite facial nerve affection; definite affection of the inferior maxillary branch of the fifth nerve and disturbed nasal function; paralysis of the sixth nérve supplying the external rectus. This lesion progressing from the tympanum and Eustachian tube, encroaching on the seniculate ganglion and causing definite motor disturbances of the facial nerve, progressed inwards, affecting the inferior branch of the fifth nerve and then forwards and down along the Eustachian tube, appeared in the posterior nasal fossae, and traveled backwards and downwards, affecting the sixth nerve. The involvement of the other branches of the fifth was probably caused by involvement of the Gasserian ganglion. The paralysis of the tenth and eleventh cranial nerves was affected by the spreading of the growth down. 
ward and backward along the basilar process of the occipital bone. Metastases were all in the anterior triangles of the neck or in the mediastinum.

The etiologic importance of syphilis was probably nil. There was a very strong malignant inheritance.

COURSE, DIAGNUSIS AND TREATMENT.

The course of the disease was about two and a half years. The more definite period was about eighteen months. The marked symptoms lasted about six months, the grave symptoms about three months.

The case was an important one. The patient consulted eighteen specialists and received every care and attention possible.

The definite diagnosis was delayed for some time after the patient was first seen on account of his extreme horror of anything in the nature of an operation. This attitude prevented satisfactory microscopic examination of the growth. $\mathrm{He}$ utterly refused to submit to any more operating.

The long interval which elapsed between the commencement of the first symptoms of the growth and its exenteration was too great for a simple removal. At the time of operation it was far too extensive to have been eradicated by the tympanic operation. The result of the operation was a lessening of the discomfort referred to the ear and apparent cessation of the growth in the ear. This good local restilt, the author believes. was due to the post-operative use of radium.

The result of the operation suggests the possibility of a successful total extirpation if an operation could have been performed when the symptoms were first definitely noted.

The final course of the disease made it clear that an exten:sive radical operation would have made the last days of the patient more comfortable, if not more numerous. Unfortunately, however, he would not submit to it. 